\title{
Total phenolic content and antioxidant activity of rice wine from waxy pigmented and non-pigmented rice varieties produced by traditional and multi-parallel fermentation
}

\author{
${ }^{1,2 *}$ Chay, C., ${ }^{2}$ Dizon, E.I., ${ }^{3}$ Hurtada, W.A., ${ }^{4}$ Elegado, F.B., ${ }^{1}$ Norng, C. and ${ }^{2}$ Raymundo, L.C. \\ ${ }^{1}$ Royal University of Agriculture, Phnom Penh, Cambodia \\ ${ }^{2}$ Institute of Food Science and Technology, College of Agriculture and Food Science, University of the \\ Philippines Los Baños, College, Laguna - 4031, Philippines \\ ${ }^{3}$ Institute of Human Nutrition and Food, College of Human Ecology, University of the Philippines Los \\ Baños, College, Laguna - 4031, Philippines \\ ${ }^{4}$ The National Institute of Molecular Biology and Biotechnology (BIOTECH), University of the Philippines \\ Los Baños, College, Laguna - 4031, Philippines
}

\begin{abstract}
Article history:
Received: 2 February 2019

Received in revised form: 4 September 2019

Accepted: 6 September 2019

Available Online: 16

September 2019
\end{abstract}

\section{Keywords:}

Total phenolic content,

Antioxidant activity,

Rice wine,

Traditional,

Multi-parallel fermentation

DOI:

https://doi.org/10.26656/fr.2017.4(1).036

\begin{abstract}
The study determined the total phenolic (TP) content, antioxidant activity (AOA) and copper reduction antioxidant capacity (CUPRAC) of rice wine from waxy pigmented and non-pigmented rice varieties using traditional and multi-parallel fermentation methods with different starter cultures. The rice wine produced using waxy pigmented rice had high TP and AOA compared to rice wine produced using waxy non-pigmented rice regardless of the method of fermentation (traditional or multi-parallel). The type of starter culture had less effect on the TP, AOA and CUPRAC of the rice wines. The results of the study revealed that the rice wine produced using the Cambodian waxy rice possess bioactive compounds that could function as antioxidants, with higher values obtained from pigmented than the non-pigmented variety. Hence, this study proved that rice wine is a healthy alcoholic drink. However, future research should be conducted to identify the specific antioxidant compounds in rice wine using waxy pigmented rice.
\end{abstract}

\section{Introduction}

Rice wine is a traditional type of alcoholic beverage made from rice through the process of fermentation. Drinking too much rice wine, or any other alcoholic beverage has some negative side effects in the body such as nausea, hangover and blurry vision, loss of balance and muscle control. However, moderate drinking of wine has some health benefits. It was reported to be a potential protectant from UV-induced skin aging phenomena. Seo et al. (2009) reported that when fibroblasts (cells of connective tissue) were treated with rice wine, the expression of pro-collagen increased. In addition, rice wine has also been linked to promoting better blood circulation and enhanced body metabolism. There are citric and lactic acids in rice wine which helps in the digestion of the food (Rucker, 2007). When food is properly digested, nutrients are better sorted out and transferred to the proper body organ. There were also studies that specially brewed medicinal rice wine can have more beneficial effects than other regular wines. Rice wine has also been considered to be healthier than wine from grapes because it contains large amount of protein, sugar and vitamin B2 (Benefits of rice wine, 2016). These factors have been shown to regulate blood sugar, plus vitamin B2 that supports the liver giving it more energy to assist with alcohol digestion.

A 70-year old woman, Singha Towit, has been making rice wine in Pursat province, Cambodia for 30 years. She claims that a glass of rice wine gives her the energy to put in a full day's hard work without feeling exhausted, despite her age. She said "Everyone should drink a small glass or two each day", she says. "It is good for your body and good for your skin."

Indeed, rice and herbal wine concoctions are believed to cure many ailments, according to winemakers and regular drinkers. Rice wines give energy and help build a strong body. In the countryside, a glass of rice wine is commonly drunk each morning after breakfast to induce energy for the day ahead. After childbirth many Khmer women drink it mixed with honey and fruit to restore their health, prevent food poisoning and to warm their bodies. Towit recommends that a postnatal woman drink it regularly until she is 
confident her body has recovered (Shelton, 2007).

In recent years, there has been an increased awareness on the effect of food on health, thus leading to a rise in the consumption of fruit, vegetables, and cerealbased food. Phenolic compounds have received considerable attention because their dietary intake is related to lower incidence of chronic degenerative diseases, such as cancer, diabetes, Alzheimer's disease and cardiovascular diseases. Cereals, fruits, and vegetables are rich sources of phenolic compounds. In fact, the health benefits of their dietary intake have been related, at least in part, to their phenolic compounds content. Phenolic compounds are among the healthpromoting phytochemicals present in cereals and are receiving much attention because of their antioxidant properties. It has been reported that phenolic compounds play a significant role in the prevention of many chronic diseases due to their antioxidant, anti-inflammatory and anti-carcinogenic properties (Bondia-Pons et al., 2009).

The polyphenolic compounds in wines are beneficial to human health. Chinese rice wine was found to contain various phenolic compounds with $(+)$-catechin and syringic acid being the dominant ones, which are highly correlated with the antioxidant activities in the rice wines that were tested (Que, Mao and Pan, 2006). In a study by Teramoto et al. (1994), sake made from purple rice wine was found to have high phenolic and anthocyanin content which are compounds with high antioxidant properties. Anthocyanins are polyphenolic compounds that have been found to exhibit four times greater antioxidant capacity than ascorbic acid (Zhang et al., 2010).

Antioxidants are substances that can prevent or slow damage to cells caused by free radicals, unstable molecules that the body produces as a reaction to environmental and other pressures. Antioxidant compounds act by scavenging radicals known as reactive oxygen species (ROS). The consumption of wine may help alleviate the effects of ROS and thus be ultimately beneficial for consumers (Que, Mao and Pan, 2006; Bandonill et al., 2009).

Rice wine is a traditional alcoholic beverage in many parts of Asia. It is produced by microbial fermentation of steamed rice with yeast and water. Different versions of this drink exist and they are locally known by different names; for instance: sake in Japan, makgeolli or takju in Korea, tapuy in the Philippines and brem in Bali. Although rice wine has been introduced to many other countries through trade and globalization, research into its characteristics and health benefits is still predominantly conducted in the regions of its origin (Rucker, 2007). In Cambodia, however, there were no reports available on the bioactive compounds present in the local rice wine produced through the traditional method.

Thus, this study evaluated the total phenolic content, copper reducing activity and antioxidant activity of the rice wine made from waxy pigmented and nonpigmented Cambodian rice varieties produced through traditional and multi-parallel fermentation.

\section{Materials and methods}

\subsection{Materials}

Waxy pigmented and non-pigmented rice varieties were purchased from Cambodia and transported to the Philippines for rice winemaking and the total phenolic content and antioxidant activity of the products were analyzed. Waxy pigmented rice known as angkordomneubkhmao or black sticky rice was obtained from Trapeang Ta Moung Village, Prey Sleok Commune, Treang District, Takeo Province. The waxy non-pigmented rice, locally known as angkordomneubsor or white sticky rice, was collected from Teamchas Village, Kompongsvay Commune and District, Kompong Thom Province, Cambodia.

Pure culture of molds and yeasts namely, Mucor spp., Rhizopus oryzae, Saccharomycopsis spp., Candida tropicalis, and Saccharomyces cerevisiae were utilized as inoculants for the preparation of the instant starter culture in the laboratory (developed starter culture). These microorganisms were isolated from medombae (dried starter culture) of Kampong Cham province in the previous work of Chim et al. (2014). Aside from the developed starter culture, a commercial medombae purchased in the local market was used as inoculants in the fermentation of rice wine.

\subsection{Development of dried starter culture}

Microbial strains found to have an important role in rice wine fermentation were selected and incorporated in the preparation of instant/dried starter culture (Figure 1). The optimized temperature and time of drying, moisture content and other factors affecting the viability of microorganisms in ground rice during starter were applied based on the previous studies of Dizon et al. (2009). Figure 2 shows the dried starter cultures utilized in the study.

After preparation, drying and cooling of the medombae starter cultures, they were packed in $\mathrm{PE}$ plastic bag and were stored at refrigerated temperature (5 $\left.-8^{\circ} \mathrm{C}\right)$. 


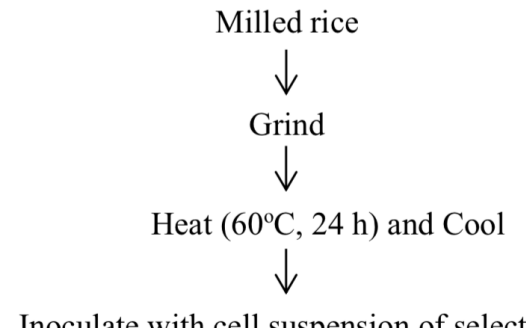

Inoculate with cell suspension of selected mold and yeast isolates

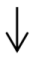

Add appropriate amount of sterilized distilled water

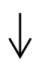

Mould into desired shape

$\downarrow$

Store at room temperature for $24 \mathrm{~h}$

$\downarrow$

${ }^{a}$ Dry in an oven at $37^{\circ} \mathrm{C}$

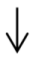

Dried Starter Culture

(Developed in the Laboratory)

${ }^{a}$ Repeat the process until completely dried

Figure 1. Preparation of dried starter culture inoculated with selected mold and yeast isolates.
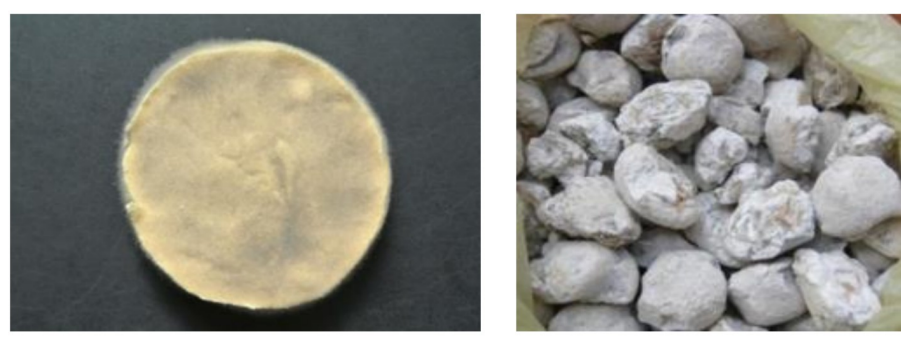

Dried Instant Starter

Culture (Developed)

Commercial Dried Starter Culture (Medombae)

Figure 2. Dried starter cultures used in rice wine fermentation (traditional and multi-parallel) using wax pigmented and nonpigmented rice varieties.

\subsection{Rice wine fermentation}

\subsubsection{Traditional method.}

The traditional process for making rice wine at laboratory scale was done. A total of $2 \mathrm{~kg}$ of each of waxy pigmented and non-pigmented rice were soaked in water for $2 \mathrm{hrs}$, drained and steamed for $60 \mathrm{mins}$ and cooled. Then, $1 \%$ of the developed starter culture and commercial medombae were inoculated separately in steamed rice. The mixtures were placed in gallon jars for solid state fermentation ( $72 \mathrm{hrs})$, and then $2.8 \mathrm{~L}$ of distilled water was added for liquid state fermentation (2 weeks). The fermented mixtures were filtered and allowed to stand for 2 weeks at $25^{\circ} \mathrm{C}$ for sedimentation.

\subsubsection{Multi-parallel method.}

Waxy pigmented and non-pigmented rice wine processing was conducted using the multi-parallel method, separately. The method initially used a mixture of $75 \mathrm{~g}$ saccharified rice (using the dried starter culture in the laboratory and commercial medombae, as inoculants), $187.5 \mathrm{~g}$ steamed rice and $262.5 \mathrm{~mL}$ water inoculated with $1 \%$ developed starter culture. After 72 hrs, first dilution/addition was done using a mixture of $300 \mathrm{~g}$ steamed rice, $120 \mathrm{~g}$ saccharified rice, and 232.5 $\mathrm{mL}$ of water. The mixture was transferred in gallon jar with provision of enough space for the succeeding dilutions. The mash was further diluted with $600 \mathrm{~g}$ steamed rice, $180 \mathrm{~g}$ saccharified rice, $952.5 \mathrm{~mL}$ water and after one day the fermenting mash was added with $1072.5 \mathrm{~g}$ steamed rice, $255 \mathrm{~g}$ saccharified rice and $3337.5 \mathrm{~mL}$ water. After the last addition, the mixture was allowed to ferment for two weeks undisturbed in the fermentation gallon jars, separately. After fermentation, the mash (from both rice varieties) was filtered; the extracted wines were placed in clean gallon jars and stored at $25^{\circ} \mathrm{C}$ for clarification by natural sedimentation.

\subsection{Determination of total phenolic content}

The total phenolic content of rice wines was determined by Folin-Ciocalteu method following the procedure of Escribano-Bailón et al. (2002). The sample was diluted with water and then to $0.5 \mathrm{~mL}$ of the samples, $0.5 \mathrm{~mL}$ of Folin-Ciocalteu's phenol reagent (Sigma-Aldrich) and $0.5 \% \mathrm{Na}_{2} \mathrm{CO}_{3}$ were added. After standing for 5 mins, $5 \mathrm{~mL}$ distilled water was added. The absorbance readings were measured at $720 \mathrm{~nm}$ (Shimadzu UV-1601 spectrophotometer) with water plus reagent as blank samples. Total phenolic content was computed in a standard curve with gallic acid as reference phenol. The results were expressed as gallic acid equivalents $\left(\mathrm{GAE} \mathrm{mL}^{-1}\right)$.

\subsection{Determination of antioxidant activity (DPPH radical scavenging method)}

The antioxidant activity of polyphenols was measured in terms of hydrogen donating or radical scavenging ability using the stable radical 2,2-diphenyl-1 -picrylhydrazyl (DPPH) according to the method of Ribeiro et al. (2008) with some modifications, using synthetic antioxidants butylated hydroxy-anisole (BHA) and butylated hydroxy-toluene (BHT) as reference antioxidants.

A total of $5 \mathrm{~mL}$ of $0.1 \mathrm{mM}$ DPPH were pipetted in test tubes and added with $100 \mathrm{uL}$ of the standards and sample. The mixture was vortexed and allowed to stand for 20 mins. The control consisted of $100 \mathrm{uL}$ methanol and the reagent served as control. The absorbance of the solution was read at $517 \mathrm{~nm}$ against reagent blank (Shimadzu UV-1601 spectrophotometer). The 
percentage of inhibition was expressed using the following equation:

$$
\% \text { Inhibition }=\left[\frac{A_{\text {control }}-A_{\text {Sample }}}{A_{\text {Control }}}\right] \times 100
$$

\subsection{Copper reduction antioxidant capacity (CUPRAC) test}

Copper reduction antioxidant capacity (CUPRAC) test is a variant of FRAP test using $\mathrm{Cu}$ instead of Fe. The assay is based on the reduction of $\mathrm{Cu}^{+2}$ to $\mathrm{Cu}^{+1}$ by the combined action of all antioxidants (reducing agents) in a sample. The $\mathrm{Cu}^{+1}$ then forms a yellow colored complex with neocuproine (2,9-dimethyl-1,10-phenanthroline) which absorbs at $450 \mathrm{~nm}$. Therefore, the higher the absorbance at $450 \mathrm{~nm}$, the higher the reduction of copper by the antioxidants, the higher the antioxidant capacity, the better the health beneficial quality of foods. A total of $1 \mathrm{~mL}$ of Copper chloride $\left(\mathrm{CuCl}_{2}\right), 1.0 \mathrm{~mL}$ of $\mathrm{NH}_{4} \mathrm{AC}$, $1.0 \mathrm{~mL}$ of neocuproine, $0.5 \mathrm{~mL}$ of antioxidant and 0.6 $\mathrm{mL}$ of water were pipetted in test tubes. The concentration of antioxidants was prepared using different concentrations of $5,10,15,20$ and $25 \mu \mathrm{g}$ $\mathrm{GAEmL}^{-1}$. The mixture was vortexed and allowed to stand for 30 mins and then read at $450 \mathrm{~nm}$ against a blank sample consisted of water and the reagents (Alpinar et al., 2009).

\subsection{Data analysis}

Data were analyzed using Analysis of Variance (ANOVA). Samples found to be significantly different were further subjected to Duncan's New Multiple Range Test (DNMRT) to locate the differences among samples.

\section{Results and discussion}

\subsection{Total phenolic content (TP)}

Due to its antioxidant activity, the phenolic compounds provide beneficial health effects. The phenolic compounds have long been associated with a variety of biochemical and pharmacological properties including antioxidant, antiviral, anticancer, and antiinflammatory activities. In natural plants, phenolic compounds have attracted considerable attention with regard to their physiological functions in protecting human bodies against oxidation, aging, cancer, and diabetes (Xu et al., 2015). Polyphenols are the most effective antioxidative constituents in plant products consumed (Escribano-Bailon et al., 2004). Several literatures reported the health benefits of phenolics, hence the popularity of dietary intake of products including wines, rich in flavonoids and anthocyanins became popular (Podsedek, 2007). As a result, consumption of wine products also shoots up in most ASEAN countries.
The total phenolic content of rice wine made from waxy pigmented and non-pigmented Cambodian rice varieties is presented in Table 1 . It can be seen that the total polyphenol content of the different types of waxy rice wine is significantly different from each other. The total phenolic contents of rice wine from waxy pigmented rice ranged from 577.40 - $607.40 \mu \mathrm{g}$ GAE mL ${ }^{-1}$, while rice wine from waxy non-pigmented rice ranged from 478.60 - $503.60 \mu \mathrm{g}$ GAE $\mathrm{mL}^{-1}$. This is much higher than that of tapuy with 118-303 $\mu \mathrm{g} \mathrm{GAE} / \mathrm{ml}$ (Hipol and Alma-in, 2018) and takju wine with 21.40 - $77.9 \mu \mathrm{g}$ $\mathrm{GAE} / \mathrm{ml}$ (Hong et al., 2009) but lower than that of North China rice wine with $722.43 \mu \mathrm{g} \mathrm{GAE} / \mathrm{ml}$ (He et al., 2013). However, similar research study has not been reported for Cambodian rice wine.

Table 1. Total phenolic content of rice wine at varying fermentation method, starter culture and rice variety

\begin{tabular}{ccc}
\hline \multirow{2}{*}{ Rice Variety } & $\begin{array}{c}\text { Fermentation } \\
\text { Method }\end{array}$ & \begin{tabular}{c} 
Total Phenolics \\
\cline { 2 - 3 }
\end{tabular} \\
\hline \multirow{3}{*}{ Waxy pigment rice } & TD & $577.4^{\mathrm{c}}$ \\
& MD & $586.4^{\mathrm{b}}$ \\
& TM & $607.4^{\mathrm{a}}$ \\
Waxy non-pigmented rice & TD & $503.6^{\mathrm{d}}$ \\
& MD & $478.6^{\mathrm{f}}$ \\
& TM & $483.6^{\mathrm{e}}$ \\
\hline
\end{tabular}

Means within column with the same superscripts are not significantly different at $\mathrm{P} \leq 0.05$.

TD- Traditional fermentation method using "developed" starter culture;

MD-Multi-parallel fermentation method using "developed" starter culture;

TM-Traditional fermentation method using Cambodian traditional medombae starter culture

In general, rice wine produced from waxy pigmented rice obtained significantly higher total phenolic contents as compared to the products made from waxy nonpigmented rice regardless of the method of the fermentation and starter culture used. In regards to the method of fermentation, the rice wine produced using waxy pigmented rice variety by the traditional method suggests lower values $\left(577.40 \mu \mathrm{g} \mathrm{GAE} \mathrm{mL}^{-1}\right)$ than the multi-parallel method $\left(586.40 \mu \mathrm{g} \mathrm{GAE} \mathrm{mL}^{-1}\right)$. However, higher phenolic contents were observed in rice wine using waxy non-pigmented rice produced by the traditional method $\left(503.60 \mu \mathrm{g} \mathrm{GAE} \mathrm{mL}^{-1}\right)$ than the multiparallel $\left(478.60 \mu \mathrm{g} \mathrm{GAE} \mathrm{mL}^{-1}\right)$ fermentation. This can be attributed to the dilution or addition of water during rice wine processing using multi-parallel fermentation.

In this study, there are two types of starter cultures (developed/prepared in the laboratory and medombae, a traditional dried Cambodian starter) used in the fermentation of rice wine using two varieties of Cambodian rice (waxy pigmented and non-pigmented). The developed starter culture was prepared in the 
laboratory using ground rice and inoculated with pure cultures of isolated useful microorganisms from medombae prior to drying. As to the effect of the starter cultures, significant differences were observed among samples. There was no clear trend observed on values but rather the effect is more on the rice varieties used, with waxy pigmented rice had higher total phenolic contents than the waxy non-pigmented rice. In the study of Cai et al. (2019), they found out that black rice was more preferred for the optimum polyphenolic content and antioxidant activity. In addition, the results indicated that the polyphenolics of the rice wines were mainly derived from their plant materials and that various rice materials could result in drastically different wine quality. In contrast, the total polyphenol contents could be less significantly changed by using different starters. This confirms the results of this study that the wine from waxy pigmented rice has higher phenolic contents than the waxy non-pigmented rice. On the other hand, the type of starters had no significant effect on the total phenolic content of the rice wine.

\subsection{DPPH free radical scavenging activity}

The scavenging DPPH radical is a widely used method to determine antioxidant activity in a relatively short time compared with other methods (Que et al., 2006). DPPH is a stable free radical that loses its absorbance at $517 \mathrm{~nm}$ when it is reduced. The DPPH free radical scavenging activity expressed as percentage inhibition of wine samples made from waxy pigmented and non-pigmented rice by traditional and multi-parallel fermentation method using the two dried starter cultures at various total phenolic (TP) concentrations are shown in Table 2. The action of antioxidant molecules on DPPH is mainly due to their hydrogen donating ability. In the presence of an antioxidant, the purple color typical to free DPPH radical decays (Hipol, 2014). Antioxidants are of great importance in terms of reducing oxidative stress that is thought to cause damage to biological molecules (Bektas et al., 2005). The ability of rice wine to reduce DPPH was thus determined spectrophotometrically by the decrease in absorbance at $517 \mathrm{~nm}$, which is induced by antioxidants.

The percentage DPPH radical scavenging activity of the rice wine samples was observed to increase with increasing TP concentration. The highest percentage inhibition of $32.75 \%$ was observed in samples using the traditional method of fermentation and utilizing medombae in waxy pigmented rice. In the five TP concentrations, the percentage of DPPH inhibition was significantly higher in wine samples made from waxy pigmented (14.31-32.75\%) than non-pigmented (10.55$16.43 \%$ ) rice in both traditional and multi-parallel fermentation method regardless of the type of starter cultures used. The values obtained in our study still demonstrate the effective reducing capacity of the rice wine.

Hipol and Alma-in (2018) reported that the Philippines rice wine (tapuy) contains natural bioactive compounds that could function as antioxidants i.e. phenolics, flavonoids and tannins. Their data suggest that the antioxidant action of tapuy is most likely related to the hydrogen accepting capacity of its bioactive compounds.

\subsection{Copper reducing activity}

Copper reduction antioxidant capacity (CUPRAC) test is a variant of FRAP test using $\mathrm{Cu}$ instead of Fe. The assay is based on the reduction of $\mathrm{Cu}^{+2}$ to $\mathrm{Cu}^{+1}$ by the combined action of all antioxidants (reducing agents) in a sample. The $\mathrm{Cu}^{+1}$ then forms a yellow colored complex with neocuproine (2,9-dimethyl1,10-phenanthroline) which absorbs at $450 \mathrm{~nm}$. Therefore, the higher the absorbance at $450 \mathrm{~nm}$, the higher is the reduction of copper by the antioxidants; the higher the antioxidant capacity, the better the health beneficial quality of foods.

Antioxidants are compounds capable of counteracting the effects of oxidative processes in cells or exogenous systems, reacting in particular with reactive oxygen or nitrogen species (Poljšak and Fink, 2014) or with other free radicals or unstable molecules

Table 2. DPPH radical scavenging activity of rice wine at varying fermentation method, starter culture and rice variety

\begin{tabular}{|c|c|c|c|c|c|c|}
\hline \multirow{3}{*}{$\begin{array}{l}\text { Total Phenolic Concentration } \\
\text { (ug GAE } \mathrm{mL}^{-1} \text { ) }\end{array}$} & \multicolumn{6}{|c|}{ Inhibition (\%) in Waxy } \\
\hline & \multicolumn{3}{|c|}{ Pigmented Rice Wine } & \multicolumn{3}{|c|}{ Non-pigmented Rice Wine } \\
\hline & TD & MD & TM & TD & MD & TM \\
\hline 5 & $14.60^{\mathrm{a}}$ & $14.31^{\mathrm{ab}}$ & $14.51^{\mathrm{a}}$ & $10.55^{\mathrm{d}}$ & $11.16^{\mathrm{c}}$ & $11.20^{\mathrm{c}}$ \\
\hline 10 & $18.93^{\mathrm{a}}$ & $18.52^{\mathrm{b}}$ & $18.64^{\mathrm{ab}}$ & $11.53^{\mathrm{d}}$ & $12.67^{\mathrm{c}}$ & $12.63^{\mathrm{c}}$ \\
\hline 15 & $23.34^{\mathrm{b}}$ & $22.73^{c}$ & $24.04^{\mathrm{a}}$ & $12.67^{\mathrm{f}}$ & $14.15^{\mathrm{d}}$ & $13.94^{\mathrm{de}}$ \\
\hline 20 & $28.09^{\mathrm{a}}$ & $26.45^{\mathrm{b}}$ & $28.21^{\mathrm{a}}$ & $13.61^{\mathrm{d}}$ & $15.45^{\mathrm{c}}$ & $15.09^{\mathrm{c}}$ \\
\hline 25 & $31.89^{\mathrm{b}}$ & $30.66^{c}$ & $32.75^{\mathrm{a}}$ & $14.23^{\mathrm{f}}$ & $16.43^{\mathrm{d}}$ & $16.11^{\mathrm{e}}$ \\
\hline
\end{tabular}

Means within column with the same superscripts are not significantly different at $\mathrm{P} \leq 0.05$.

TD- Traditional fermentation method using "developed" starter culture;

MD-Multi-parallel fermentation method using "developed" starter culture;

TM-Traditional fermentation method using Cambodian traditional medombae starter culture 
Table 3. Copper reducing activity of rice wine at varying fermentation method, starter culture and rice variety

\begin{tabular}{ccccccc}
\hline \multirow{2}{*}{$\begin{array}{c}\text { Total Phenolic Concentration } \\
\text { (ug GAE mL-1) }\end{array}$} & \multicolumn{5}{c}{ Copper Reducing Activity (Absorbance) } \\
\cline { 2 - 7 } & TD & MD & TM & TD & MD & TM \\
\cline { 2 - 6 } & $0.063^{\mathrm{a}}$ & $0.062^{\mathrm{a}}$ & $0.063^{\mathrm{a}}$ & $0.047^{\mathrm{b}}$ & $0.032^{\mathrm{c}}$ & $0.048^{\mathrm{b}}$ \\
5 & $0.117^{\mathrm{b}}$ & $0.122^{\mathrm{a}}$ & $0.123^{\mathrm{a}}$ & $0.077^{\mathrm{d}}$ & $0.066^{\mathrm{e}}$ & $0.088^{\mathrm{c}}$ \\
10 & $0.173^{\mathrm{c}}$ & $0.178^{\mathrm{b}}$ & $0.184^{\mathrm{a}}$ & $0.111^{\mathrm{e}}$ & $0.092^{\mathrm{f}}$ & $0.125^{\mathrm{d}}$ \\
20 & $0.236^{\mathrm{b}}$ & $0.232^{\mathrm{c}}$ & $0.243^{\mathrm{a}}$ & $0.132^{\mathrm{e}}$ & $0.118^{\mathrm{f}}$ & $0.160^{\mathrm{d}}$ \\
25 & $0.290^{\mathrm{b}}$ & $0.284^{\mathrm{c}}$ & $0.294^{\mathrm{a}}$ & $0.159^{\mathrm{e}}$ & $0.138^{\mathrm{f}}$ & $0.196^{\mathrm{d}}$ \\
\hline
\end{tabular}

Means within column with the same superscripts are not significantly different at $\mathrm{P} \leq 0.05$.

TD- Traditional fermentation method using "developed" starter culture;

MD-Multi-parallel fermentation method using "developed” starter culture;

TM-Traditional fermentation method using Cambodian traditional medombae starter culture

generated during normal metabolic oxidative reactions (Benzie, 2003). Antioxidant capacity is a general capability of organisms or compounds to engage with free radicals and prevent their harmful effect (Huang et al., 2005). A very reliable method of determining the total antioxidant capacity is by using the redox system of copper (II)-neocuproine complex (copper-reducing antioxidant capacity method or CUPRAC) (Özyürek et al., 2011).

Reactive oxygen species (ROS) that emerge as a result of the respirative cycle of oxidative phosphorylation may attack biological macromolecules like cellular DNA and proteins (Apak et al., 2008). Excessive ROS may give rise to single- and doublestrand DNA breaks that may eventually cause cell aging, cardiovascular diseases, mutagenic changes and cancerous tumor growth (Halliwell and Gutteridge, 1989; Halliwell and Aruoma, 1991). Consumption of foods naturally bearing antioxidant power is the most efficient way of combating such undesired transformations and health risks. Consequently, the opportunity for improving health by improving diet is great (Ames et al. 1993).

The copper reducing activity of wine samples (Absorbance $=450 \mathrm{~nm}$ ) made from waxy pigmented and non-pigmented rice by traditional and multi-parallel fermentation method using different starter cultures at various TP concentrations (ug GAE $\mathrm{mL}^{-1}$ ) are shown in Table 3. The results revealed that the copper reducing activity of rice wine samples increased with increasing TP concentration. In general, the rice wine samples made from waxy pigmented rice variety have significantly higher values than the waxy non-pigmented rice. At the highest TP concentration of 25 ug GAE mL ${ }^{1}$, the rice wine from pigmented rice and fermented by the traditional method using commercial starter culture (medombae) as inoculant had the highest value of $0.294 \%$. This is followed by rice wine $(0.290 \%)$ also from waxy pigmented rice and fermented by traditional method using the starter culture prepared in the laboratory ("developed"). The lowest value was observed in samples using multi-parallel fermentation method $(0.284 \%)$. On the other hand, rice wine from waxy non-pigmented rice obtained copper reducing activity which ranged from $0.196-0.138 \%$. The lower values recorded for the multi-parallel method of fermentation for both rice varieties can be attributed to the dilution effect of water as part of the processing.

In the present study, total phenolic content, copper reducing activity and antioxidant activity were found higher in rice wine made from waxy pigmented rice than from waxy non-pigmented rice regardless of the method of fermentation and starter culture used.

\section{Conclusion}

The rice wine produced using waxy pigmented rice has high TP, DPPH, and CUPRAC compared to rice wine produced using waxy non-pigmented rice regardless of the method of fermentation and starter culture used. The results of the study revealed that the rice wine produced using the Cambodian waxy pigmented rice and the traditional method of fermentation with commercial medombae (dried starter) or prepared in the laboratory (developed starter) possess antioxidant properties. Hence, this study proved that rice wine is a healthy alcoholic drink. Active promotion and introduction of locally produced rice wine for commercialization will undoubtedly help or minimize most health problems of the consumers and at the same time boost the economy of the country. It is however recommended that future research be done on the specific antioxidant compounds present in Cambodian rice wine and wine produced using the methods in this study.

\section{Acknowledgments}

The authors extend their gratitude and appreciation to the Southeast Asian Regional Center for Graduate Study and Research in Agriculture (SEARCA), Philippines for the scholarship grant; Royal University of Agriculture (RUA), for providing the opportunity and 
valuable assistance for the completion of the study; Institute of Food Science and Technology, and BIOTECH (UP Los Baños) laboratories, Philippines for their assistance in the determination of the total phenolic content and antioxidant activity of the rice wine samples.

\section{References}

Alpinar, K., Ozyurek, M., Kolak, U., Guclu, K., Aras, M., Altun. C., Celik, S.E., Berker, K.I., Bektasoglu, B. and Apak, R. (2009). Antioxidant capacities of some food plants wildly grown in Ayvalik of Turkey. Food Science Technology Research, 15(1), 59-64. https://doi.org/10.3136/fstr.15.59

Ames, B.N., Shigenaga, M.K. and Hagen, T.M. (1993). Oxidants, antioxidants, and degenerative diseases of aging. Proceedings of the National Academy of Sciences of the United States of America, 90(17), 7915-7922. https://doi.org/10.1073/pnas.90.17.7915

Apak, R. Güçlü, K., Özyürek, M. and Çelik, S.E. (2008). Mechanism of antioxidant capacity assays and the CUPRAC (cupric ion reducing antioxidant capacity) assay. Microchim Acta, 160(4), 413-419. https:// doi.org/10.1007/s00604-007-0777-0

Bandonill, E.H., Morales, A.V., Ablaza, M.J., Mamucod, H.F. and Romero, M.V. (2009). Quality improvement and shelf-life enhancement of Philippine tapuy (rice wine). Philippine Journal of Crop Science, 34(1), 12- 18.

Bektas, T., Sokmen, M., Akpulat, H.A. and Sokmen, A. (2005). Use of a free radical method to evaluate antioxidant activity. Food Chemistry, 92(1), 89-92. https://doi.org/10.1016/j.foodchem.2004.07.016

Benefits of rice wine. (2016). Retrieved from website: https://studymoose.com/benefits-of-rice-wine-essay

Benzie, I.F.F. (2003). Evolution of dietary antioxidants, Comparative Biochemistry and Physiology Part A: Molecular and Integrative Physiology, 136(1), 113-2022. https:// doi.org/10.1016/S1095-6433(02)00368-9

Bondia-Pons, I., Aura, A-M., Vuorela, S., Kolehmainen, M., Mykkänen, H. and Poutanen, K. (2009). Rye phenolics in nutrition and health. Journal of Cereal Science, 49(3), 323-36. https://doi.org/10.1016/ j.jcs.2009.01.007

Cai, H., Shen, L., Zhang, Q. and Luo. J. (2019). Phenolic profile and antioxidant activity of Chinese rice wine fermented with different rice materials and starters. $L W T, 111,226-234$. https://doi.org/10.1016/ j.lwt.2019.05.003

Chim, C., Dizon, E.I., Raymundo, L.C., Elegado, F.B. and Hurtada, W.A. (2014). Quality of Rice
Wine Using Isolated Microorganisms From "Medombae," A Starter Culture from Cambodia, p. 151. Los Baños, Philippines: The University of the Philippines, $\mathrm{PhD}$ Thesis.

Dizon, E.I., Del Rosario, O. M., Romero, M.V., Bandonill, E.H. and Valerio, A.M. (2009). Standardization of starter culture for rice wine (tapuy) processing. Terminal Report. UPLBPHILRICE Joint Research Project. Los Banos, Laguna, Philippines: University of the Philippines.

Escribano-Bailón, M.T., Santos-Buelga, C. and RivasGonzalo, J.C. (2002). Anthocyanins in cereals. Journal of Chromatography A, 1054(1-2), 129-141. https://doi.org/10.1016/j.chroma.2004.08.152

Halliwell, B. and Gutteridge, J.M.C. (1989). Free radicals in biology and medicine. Oxford, UK: Oxford University Press.

Halliwell, B. and Aruoma, O.I. (1991). DNA damage by oxygen-derived species: its mechanisms and measurement in mammalian systems. FEBS Letter 281(1-2), 9-19. https://doi.org/10.1016/0014-5793 (91)80347-6

He, S., Mao, X., Liu, P., Lin, H., Du, Z., Lu, N., Han, J. and Qiu, C. (2013). Research into the functional components and antioxidant activities of North China rice wine (Ji Mo Lao Jiu). Food Science and Nutrition, 1(4), 307-314. https://doi.org/10.1002/ fsn3.39

Hipol, R. (2014). Antioxidant potentials of culturable endophytic yeasts from Phragmites australis Cav. (Trin) ex Steud. from copper-contaminated mining site in Mankayan, Benguet. Philippine Science Letters, 7(2), 337-346.

Hipol, R.L.B. and Alma-in, A.B. (2018). Antioxidant potentials of indigenously produced Benguet tapuy (rice wine). International Food Research Journal, 25(5), 1968-1976.

Hong, Y.H., Bae, S.H., Jung, E.Y., Son, H., Shin, K.S., Kwon, K.H. and Suh, H.J. (2009). Radical scavenging activities of Korean traditional rice wine, takju. Journal of Food Science and Nutrition, 14, 109-115. https://doi.org/10.3746/jfn.2009.14.2.109

Huang, D., Ou, B. and Prior, R.L. (2005). The chemistry behind antioxidant capacity assays. Journal of Agricultural and Food Chemistry, 53(6), 18411856. https://doi.org/10.1021/jf030723c

Özyürek, M., Güçlü, K. and Apak R. (2011). The main and modified CUPRAC methods of antioxidant measurement. Trends in Analytical Chemistry, 30(4), 652-664. https://doi.org/10.1016/j.trac.2010.11.016

Podsedek, A. (2007). Natural antioxidants and antioxidant capacity of Brassica vegetables: A 
review. LWT - Food Science and Technology, 40(1), 1-11. https://doi.org/10.1016/j.lwt.2005.07.023

Poljšak, B. and Fink, R. (2014). The protective role of antioxidants in the defence against ROS/RNSmediated environmental pollution. Oxidative Medicine and Cellular Longevity, 2014, 1-22. https:// doi.org/10.1155/2014/671539

Que, F., Mao, L., Zhu, C. and Xie, G. (2006). Antioxidant properties of Chinese yellow wine, its concentrate and volatiles. LWT - Food Science and Technology, 39(2), 111-117. https://doi.org/10.1016/ j.lwt.2005.01.001

Que, F., Mao, L. and Pan, X. (2006). Antioxidant activities of five Chinese rice wines and the involvement of phenolic compounds. Food Research International, 39, 581-587. https://doi.org/10.1016/ j.foodres.2005.12.001

Ribeiro, S.M.R., Barbosa, L.C.A., Queiroz, J.H., Knodler, M. and Schieiber, A. (2008). Phenolic compounds and antioxidant capacity of Brazilian mango. Food Chemistry, 110, 620-626. https:// doi.org/10.1016/j.foodchem.2008.02.067

Rucker, M. (2007). Rice wine health benefits and its effects on the body. Retrieved from Michael Rucker website: https://michaelrucker.com/functionalsupplements/rice-wine-health-benefits/.

Seo, M., Chung, S., Choi, W., Seo, Y., Jung, S., Park, J. and Park, C. (2009). Anti-aging effect of rice wine in cultured human fibroblasts and keratinocytes. Journal of Bioscience and Bioengineering, 107(3), 266-271. https:// doi.org/10.1016/j.jbiosc.2008.11.016

Shelton, T. (2007). Rice wine believed to cure many ills. Retrieved from The Phnom Penh Post website: http://www.phnompenhpost.com/national/rice-winebelieved-cure-many-ills.

Teramoto, Y., Saigusa, N., Ueda, S. and Yoshizawa, K. (1994). Production and characteristics of red rice sake. Journal of the Institute of Brewing, 100, 3-6. https://doi.org/10.1002/j.2050-0416.1994.tb00802.x

Xu, E.B., Wu, Z.Z., Long, J., Wang, F., Xu, X.M., Jin, Z.Y. and Jiao, A.Q. (2015). Improved bioaccessibility of phenolics and antioxidant activity of glutinous rice and its fermented Chinese rice wine by simultaneous extrusion and enzymatic hydrolysis. Journal of Functional Foods, 17, 214-226. https:// doi.org/10.1016/j.jff.2015.05.032

Zhang, K., Yu, H., Shi, K., Zhou, Y., Yu, J. and Xia, X. (2010). Photoprotective roles of anthocyanins in Begonia semperflorens. Plant Science, 179, 202208. https://doi.org/10.1016/j.plantsci.2010.05.006 